\title{
Risk of peritoneal dissemination in stomach cancer
}

\author{
Robert Sitarz ${ }^{1,2 *}$, Wojciech P Polkowski ${ }^{3}$, \\ Ryszard Maciejewski ${ }^{2}$, G Johan A Offerhaus ${ }^{1}$
}

${ }^{1}$ Department of Pathology, University Medical Center Utrecht, The Netherlands
2 Department of Anatomy, Medical University of Lublin, Jaczewskiego 4, 20-090 Lublin, Poland
${ }^{3}$ Department of Surgical Oncology, Medical University of Lublin, Poland

\begin{tabular}{|c|c|}
\hline ARTICLE INFO & ABSTRACT \\
\hline $\begin{array}{l}\text { Received } 27 \text { September } 2017 \\
\text { Accepted } 29 \text { December } 2017\end{array}$ & $\begin{array}{l}\text { With regard to gastric cancer, an important disease and a public health problem, it is } \\
\text { expected that understanding the molecular make up of carcinomas will provide us with }\end{array}$ \\
\hline $\begin{array}{l}\text { Keywords: } \\
\text { gastric cancer, } \\
\text { peritoneal dissemination, } \\
\text { biomarkers, } \\
\text { risk profile, } \\
\text { immunohistochemistry. }\end{array}$ & $\begin{array}{l}\text { more precise targets for therapy. Indeed advanced molecular technology has made it } \\
\text { possible to classify according to genotype instead of phenotype. For advanced stomach } \\
\text { cancer, however, surgery is still the only option for cure. Yet, also after surgery, more } \\
\text { than } 50 \% \text { of the patients will die of peritoneal dissemination of their disease. This review } \\
\text { looks at the molecular mechanism of peritoneal spread of stomach cancer in order to } \\
\text { arrive at a risk profile that enables medical personnel to raise the index of suspicion for } \\
\text { peritoneal carcinomatosis. The peritoneal cancer index (PCI) provides a scoring system } \\
\text { to measure the extent of peritoneal spread during laparoscopic staging. A recently } \\
\text { developed device called the 'MacSpec pen' maybe of use to confirm the presence of tumor } \\
\text { when there is doubt about the diagnosis. Treatment of peritoneal dissemination consists } \\
\text { of cytoreduction, combined with hyperthermic peritoneal chemotherapy (HIPEC). }\end{array}$ \\
\hline
\end{tabular}

\section{INTRODUCTION}

In today's world, stomach cancer is an important disease and public health problem. Worldwide, it is the second most frequent cause of death due to cancer. There are, however, considerable geographical differences in the incidence and prevalence of gastric cancer. In the Western world, gastric cancer incidence has declined significantly over the last decades; high risk areas are mostly found at the eastern part of the globe [1].

Traditionally, stomach cancer was subdivided in two types: the intestinal type of stomach cancer and the diffuse type of stomach cancer. The diffuse type of stomach cancer is characterized by a discohesive growth pattern of loosely arranged tumor cells (frequently, but not necessarily, signet ring cells), and the lack of easily detectable precursor lesions such as intestinal metaplasia and dysplasia. Its incidence is pretty stable worldwide and it does not show a secular trend. This contrasts with the intestinal type of gastric cancer. The intestinal type of stomach cancer has typically a high incidence in high risk areas. It grows in tubular structures, and it is found in the gastric mucosa that shows intestinal metaplasia. Moreover, it is preceded by low- and high-grade

\footnotetext{
* Corresponding author

e-mail: r.sitarz@umlub.pl
}

dysplasia, respectively. It is felt that the diffuse type is, in large part, genetically determined, whereas the intestinal type is mostly caused by environmental factors. The partition in diffuse and intestinal type of stomach cancer is known as the so-called 'Lauren classification' [2].

The microorganism Helicobacter pylori (H. pylori), discovered by Marshall and Warren in 1983, is a first class carcinogen for both types of stomach cancer [3].

\section{MOLECULAR PATHOLOGY OF STOMACH CANCER}

Cancer is a disease of the genes. An interplay of mutated oncogenes, tumor suppressor genes and DNA repair genes induces the increasing genetic instability that leads to the aberrant cell growth and the stepwise progression to cancer and metastasis [4]. Different genotypes may be accompanied by different phenotypes, but it is believed that the aberrant genetics provide better targets for therapy, and, thus, there is a trend to subdivide and classify solid tumors based on their genetic make-up, instead of their phenotypic appearance. This also holds true for stomach cancer. In recent years, cancer specialists have proposed subdividing stomach cancers based on their molecular genetic alterations. Interestingly, these molecular classifications correspond to a large 
extent with the Lauren classification. Molecular-based pathology is, hence, rather a fine tuning of the previous Lauren classification than a complete deviation from this bipartite [5].

The prototype of the diffuse type gastric cancer is hereditary diffuse gastric cancer (HDGC). It is caused by a germline mutation in $\mathrm{CDH} 1$, a gene that encodes for E-cadherin. HDGC is an autosomal dominant disease, and carriers of the mutation have a more than $80 \%$ risk of stomach cancer at a young age. The disease is, not surprisingly, also associated with an increased risk of lobular breast cancer. Prophylactic gastrectomy is the only measure to avoid stomach cancer. E-cadherin is a cell adhesion molecule that forms a complex with catenins, and in this fashion, it is also connected with Wnt signalling. Infection with $H$. pylori can cause hypermethylation of the promotor region of $\mathrm{CDH} 1$ and silencing of the expression of E-cadherin.

The intestinal type of gastric cancer is the end result of a cascade that starts with chronic atrophic gastritis, regularly initiated by an $H$. pylori gastritis. The corpus predominant H. pylori gastritis, in particular, is a risk factor for stomach cancer. Intestinal metaplasia and dysplasia are precursor lesions of this type of stomach cancer. The tumor suppressor gene TP53 is mutated in more than $50 \%$ of these cancers.

Special forms of stomach cancer are the Epstein-Barr virus (EBV) positive stomach cancers and the gastric cancers with a defect in the mismatch repair genes. Still, by far, the majority of prevalent stomach cancers comprise the first two mentioned diffuse and intestinal types of stomach cancer.

\section{PERITONEAL DISSEMINATION OF GASTRIC CANCER}

Surgery is the only option for curing advanced gastric cancer. Nevertheless, more than $50 \%$ of patients with advanced stomach cancer will ultimately die from peritoneal dissemination of their stomach cancer [6]. CEA measurements in washings of the abdominal cavity, as well as cytology maybe good biomarkers for the presence of peritoneal metastases. Cytoreduction, combined with hyperthermic intraperitoneal chemotherapy, is, in the case of peritoneal spread, an approach that is applied nowadays, and its success varies. A better approach would be to determine which patients carry an increased risk of peritoneal disease ahead of time because it would potentially enable the taking of preventive measures that could be more successful. In order to construct a risk profile for the individual patient operated upon for stomach cancer, it is important to understand the different steps leading to peritoneal metastases and to dissect the molecular factors that play a role in the dissemination to the peritoneum.

\section{PATHOGENESIS OF PERITONEAL SPREAD OF STOMACH CANCER}

Various steps can be distinguished in the pathogenesis of metastasis of stomach cancer to the peritoneum. Firstly, cells have to detach from the original tumor and stomach wall. They then have to survive in the abdominal cavity thereafter. The next step is the attachment to the peritoneum, and then they have to create a niche and activate neovascularisation to outgrow into a metastasis [7]. The cell adhesion molecule E-cadherin is an important player in the cohesiveness of stomach cancers, as mentioned earlier. Loss of function of $\mathrm{CDH} 1$, the gene that encodes E-cadherin, leads to diffusely invading tumor cells that can easily penetrate the gastric wall and spread into the abdominal cavity. The CDH1 loss of function is brought about by mutation, loss of heterozygosity (LOH) or hypermethylation of the promotor region of the gene. As a consequence, one will observe either abnormal expression or complete absence of expression when looking at the immunohistochemical staining for this protein on a section slide of the stomach cancer (in such situations, the normal membranous staining is lost).

Once the tumor cells reach the intraperitoneal space, they must survive under relatively hypoxic conditions. Hypoxiainducible factor-1 alfa (HIF-1a) is a transcription factor that regulates cellular response to hypoxia. Overexpression of HIF-1a is associated with an increased risk of peritoneal metastasis [8], and it is overexpressed in many solid tumors and can be visualised by immunohistochemistry. Another factor considered important for survival of the tumor cells is the tumor suppressor gene PTEN. It is located on chromosome 10q, and its function is lost in many solid tumors. Overexpression or knock down of PTEN leads to either inhibition of adhesion and migration of gastric cancer cells, or the opposite, respectively, and can in this way facilitate the peritoneal dissemination [9]. When there is loss of PTEN, immunohistochemistry for this protein will show complete absence of staining.

The epidermal growth factor family is a group of receptor tyrosine kinases that includes Her2Neu. Proliferation and migration through the peritoneal cavity can be promoted by this class of growth factors. Her2Neu expression in gastric tumor cells can be visualized using immunohistochemistry. Her2Neu is a therapeutic target in stomach cancer using monoclonal antibodies that compete with the regular ligands. Overexpression through amplification of the receptor can be confirmed with in situ hybridization with a probe specifically directed against the molecule; this will also distinguish lowand high-level amplification. Importantly, grading of the overexpression in stomach cancer differs from the system used in breast cancer and follows the rules of the TOGA trial [10].

Connective tissue growth factor (CTGF) is a secretory protein that promotes cellular growth and angiogenesis by promoting endothelial cell proliferation. Interestingly, overexpression of CTGF is common in certain carcinomas, such as pancreatic and gastric cancer, but it inhibits peritoneal spread of stomach cancer. It binds to alfa3-beta1 integrin, a cell adhesion molecule that facilitates homing and binding to the mesothelial cells of the peritoneum. Stable CTGF transfectants diminished the number of peritoneal implants in mice [11].

Matrix metalloproteinases (MMPs) are a family of proteolytic enzymes capable of degradation of the various components of the extracellular matrix. MMP7 is the most potent proteolytic enzyme, and it activates other members of this family. Antisense oligonucleotides specific for MMP7 inhibit the invasiveness of gastric cancer cells into the peritoneum 
[12]. MMP7 also induces epithelial to mesenchymal transformation, thereby increasing the invasive potential of individual tumor cells.

Finally, once cells have degraded the basement membrane and invaded the peritoneum, angiogenesis is critical for the oxygenation of the tumor cells. Vascular endothelial growth factor (VEGF) secreted by the cancer cells promotes neovascularisation, and it increases the accessibility of the vascular wall for tumor cells. Antibodies specifically binding to the VEGF receptor inhibit peritoneal seeding and increase survival [13].

\section{RISK PROFILE FOR PERITONEAL DISSEMINATION IN STOMACH CANCER}

Upon summarizing the previously mentioned risk factors for the peritoneal spread of stomach cancer, one could speculate about a risk profile for peritoneal dissemination that could be evaluated based on features of the primary tumor. This could potentially increase the index of suspicion and it is conceivable that in that case preventive measures would be in place.

Diffuse growing gastric cancers seem to have a higher risk - especially the ones with aberrant expression of E-cadherin. High expression of HIF-1a is also associated with higher risk, since it makes tumors more resistant against hypoxic conditions. The same is true for loss of expression of the tumor suppressor gene PTEN. High expression of CTGF, on the contrary, protects against peritoneal dissemination. Moreover, high expression of Her2Neu, MMP7 and VEGF increases the risk of implants into the peritoneum.

Treatment of peritoneal metastasis is based on cytoreduction and hyperthermic peritoneal chemotherapy. The peritoneal cancer index (PCI) provides a scoring system to determine the extent and degree of peritoneal spread based on location, number and size of the implants during laparoscopic staging [14]. Generally, a frozen section is needed to confirm the diagnosis of cancer when the surgeon is in doubt. A recent study reported on a device that makes detection of carcinoma much easier and faster, and that is applicable in the operating room next to the patient. The so-called 'MacSpec Pen' has a sensitivity, specificity and accuracy of more than $96 \%$ [15].

\section{REFERENCES}

1. IACR. World Cancer Report 2014. Stewart BW, Wild CP, editors. Lyon: World Health Organization; February 2014.

2. Lauren P. The Two Histological Main Types of Gastric Carcinoma: Diffuse and So-Called Intestinal-Type Carcinoma. An Attempt at a Histo-Clinical Classification. Acta Pathol Microbiol Scand. 1965;64:31-49.

3. Warren JR, Marshall B. Unidentified curved bacilli on gastric epithelium in active chronic gastritis. Lancet. 1983;1(8336):1273-5.

4. Vogelstein B, Kinzler KW. Cancer genes and the pathways they control. Nat Med. 2004;10(8):789-99.

5. Setia N, Agoston AT, Han HS, Mullen JT, Duda DG, Clark JW, et al. A protein and mRNA expression-based classification of gastric cancer. Mod Pathol. 2016;29(7):772-84.

6. Hartgrink HH, Jansen EP, van Grieken NC, van de Velde CJ. Gastric cancer. Lancet. 2009;374(9688):477-90.

7. Kanda M, Kodera Y. Molecular mechanisms of peritoneal dissemination in gastric cancer. World $\mathrm{J}$ Gastroenterol. 2016;22(30):6829-40.

8. Liu J, Geng X, Li Y. Milky spots: omental functional units and hotbeds for peritoneal cancer metastasis. Tumour Biol. 2016;37(5):5715-26.

9. Zhang LL, Liu J, Lei S, Zhang J, Zhou W, Yu HG. PTEN inhibits the invasion and metastasis of gastric cancer via downregulation of FAK expression. Cell Signal. 2014;26(5):1011-20.

10. Bang YJ, Van Cutsem E, Feyereislova A, Chung HC, Shen L, Sawaki A, et al. Trastuzumab in combination with chemotherapy versus chemotherapy alone for treatment of HER2-positive advanced gastric or gastro-oesophageal junction cancer (ToGA): a phase 3, open-label, randomised controlled trial. Lancet. 2010;376(9742):687-97.

11. Chen CN, Chang CC, Lai HS, Jeng YM, Chen CI, Chang KJ, et al. Connective tissue growth factor inhibits gastric cancer peritoneal metastasis by blocking integrin alpha3beta1-dependent adhesion. Gastric Cancer. 2015;18(3):504-15.

12. Yonemura Y, Endou Y, Fujita H, Fushida S, Bandou E, Taniguchi K, et al. Role of MMP-7 in the formation of peritoneal dissemination in gastric cancer. Gastric Cancer. 2000;3(2):63-70.

13. Javle M, Smyth EC, Chau I. Ramucirumab: successfully targeting angiogenesis in gastric cancer. Clin Cancer Res. 2014;20(23):5875-81.

14. Sugarbaker TA CD, Koslowe P, Sugarbaker PH. Patterns of spread of recurrent intraabdominal sarcoma. PH S, editor. Boston: Kluwer Academic; 1996.

15. Zhang J, Rector J, Lin JQ, Young JH, Sans M, Katta N, et al. Nondestructive tissue analysis for ex vivo and in vivo cancer diagnosis using a handheld mass spectrometry system. Sci Transl Med. 2017;9(406). 